\title{
MONITORING HEALTH BEHAVIOURS AND HEALTH STATUS IN NSW: RELEASE OFTHE ADULT HEALTH SURVEY 2003
}

\author{
Deborah Baker and Margo Eyeson-Annan \\ Centre for Epidemiology and Research \\ NSW Department of Health
}

In 2002, the NSW Department of Health, in conjunction with the 17 area health services, completed the first year of the New South Wales Continuous Health Survey, an ongoing survey that uses computer-assisted telephone interviewing. The main aims of the Continuous Health Survey are to provide detailed information on the health of the people of NSW, and to support the planning, implementation, and evaluation of health services and programs. Following on from previous health surveys conducted in 1997, 1998, and 2002, this is the fourth survey that has collected data on the health of adults in NSW. This article announces the release of the New South Wales Adult Health Survey 2003 and highlights changes in health behaviour, health status, and satisfaction with health services that occurred between 1997 and 2003 (Tables 1-4).

The content of the New South Wales Continuous Health Survey was developed in consultation with the Health Survey Program Steering Committee, area health services, other government departments, and a range of experts. The survey content covers the 8 priority areas outlined in Healthy People 2005: New Directions for Public Health in New South Wales. The survey questionnaire was translated into 5 languages: Arabic, Chinese, Greek, Italian, and Vietnamese. Data were collected on a range of health behaviours, health status, use of and satisfaction with health services, social capital, and demographic information.

The target population for the New South Wales Adult Health Survey 2003 was all NSW residents aged 16 years and over living in households with private telephones. Households were sampled using list-assisted random digit dialling. When a household was contacted, one person was randomly selected for interview. Information was collected on a total of 13088 adults.

\section{HEALTH BEHAVIOURS}

Between 1997 and 2003 the prevalence of some health behaviours changed (Table 1). The proportion of smokefree households (69.8 per cent to 82.5 per cent), and the proportion of homes with a smoke alarm or detector (58.2 per cent to 72.7 per cent) increased significantly. There was a significant decrease (42.3 per cent to 35.7 per cent) in the proportion of people who participated in any alcohol risk drinking behaviour. There was a significant decrease between 1997 and 2002 in the proportion of people who were current smokers (24.0 per cent to 21.4 per cent).
However, in 2003 the proportion increased to 22.5 per cent, which was not significantly different from the previous years.

Overall, there was a significant increase between 1997 and 2003 in the proportion of people eating the recommended daily vegetable intake (16.3 per cent to 19.3 per cent). Between 1997 and 2002, there was a significant decrease in the proportion of people who consumed reduced- or low-fat milk (45.7 per cent to 43.4 per cent). However, in 2003 the proportion increased to 44.0 per cent, which was not significantly different from the previous years.

Between 1997 and 2003, the proportion of people aged 65 years and over who were immunised against influenza in the previous 12 months increased significantly (57.1 per cent to 75.8 per cent). Similarly, between 2002 and 2003 the proportion of people aged 65 years and over who were immunised against pneumococcal disease in the last 5 years also increased significantly (39.4 per cent to 46.8 per cent).

Overall, there was a significant decrease in the proportion of people who undertook adequate physical activity in 2003 (45.0 per cent) compared to 1998 (47.6 per cent).

Several health behaviours remained unchanged. The proportion of people eating the recommended daily serves of fruit (45.8 per cent) was unchanged, as was high-risk drinking in the last 4 weeks (14.7 per cent).

In 2003, a new indicator on hand washing when preparing raw meat was reported for the first time and trends in this additional indicator will continue to be monitored.

\section{HEALTH STATUS}

Monitoring the health status of a population helps to detect emerging patterns of illness and disease and provides information to inform policy and planning of health services. There have been some changes in the health status of the population between 1997 and 2003 (Table 2).

Over the period 1997 to 2003 there were significant increases in the proportion of people who had been diagnosed with diabetes ( 4.7 per cent to 6.2 per cent), ever diagnosed with asthma (16.6 per cent to 21.0 per cent), and who were overweight or obese ( 42.2 per cent to 48.3 per cent). Between 1997 and 2002, there was also a significant increase in the proportion of people who reported high and very high physiological stress as measured by the Kessler 10 score (10.5 per cent to 12.2 per cent). However, in 2003, the proportion decreased to 11.1 per cent, which was not significantly different from previous years. 
The proportion of people who rated their health status as excellent, very good, or good declined significantly between 1997 (84.9 per cent) and 2003 (80.8 per cent). The proportion who reported all their natural teeth missing declined significantly between 1998 and 2003 (8.3 per cent to 5.8 per cent).
The only indicator of health status to remain unchanged between 1997 and 2003 was current asthma (10.3 per cent to 11.0 per cent).

For the first time, information on adult incontinence, falls in people 65 years and over, and additional health status

\section{TABLE 1}

TRENDS IN INDICATORS OF HEALTH BEHAVIOURS, BY SEX, NSW, 1997-2003

\begin{tabular}{|c|c|c|c|c|c|}
\hline Indicator & Year & Males & $(95 \% \mathrm{CI})$ & Females $(95 \% \mathrm{Cl})$ & Persons $(95 \% \mathrm{CI})$ \\
\hline \multirow[t]{4}{*}{ Alcohol risk drinking (Guideline 1) } & 1997 & 50.7 & $(49.3-52.2)$ & $34.1(32.9-35.4)$ & $42.3(41.3-43.3)$ \\
\hline & 1998 & 50.5 & $(49.0-52.1)$ & $36.2(34.9-37.5)$ & $43.2(42.2-44.2)$ \\
\hline & 2002 & 39.2 & $(37.3-41.1)$ & $29.7(28.1-31.2)$ & $34.4(33.1-35.6)$ \\
\hline & 2003 & 41.3 & $(39.4-43.2)$ & $30.3(28.9-31.8)$ & $35.7(34.5-36.9)$ \\
\hline \multirow[t]{2}{*}{ High risk drinking in the past 4 weeks } & 2002 & 16.7 & $(15.0-18.4)$ & $11.7(10.3-13.1)$ & $14.4(13.3-15.5)$ \\
\hline & 2003 & 17.9 & $(16.2-19.6)$ & $10.9 \quad(9.7-12.2)$ & $14.7(13.6-15.7)$ \\
\hline \multirow[t]{2}{*}{ Use public water as usual source of water } & 2002 & & & $81.1(79.5-82.7)$ & \\
\hline & 2003 & & & $81.1(80.2-82.0)$ & \\
\hline \multirow[t]{4}{*}{$\begin{array}{l}\text { Vaccinated against influenza in the last } 12 \\
\text { months }\end{array}$} & 1997 & 55.8 & $(52.3-59.2)$ & $58.2(55.3-61.0)$ & $57.1(54.9-59.3)$ \\
\hline & 1998 & 61.9 & $(58.5-65.3)$ & $64.5(61.8-67.2)$ & $63.3(61.2-65.5)$ \\
\hline & 2002 & 75.3 & $(72.4-78.3)$ & $75.7(73.0-78.3)$ & $75.5(73.5-77.5)$ \\
\hline & 2003 & 76.1 & $(73.0-79.1)$ & $75.6(73.2-78.1)$ & $75.8(73.9-77.8)$ \\
\hline \multirow{2}{*}{$\begin{array}{l}\text { Vaccinated against pneumococcal disease } \\
\text { in the last } 5 \text { years }\end{array}$} & מחמח & 367 & $122=1011$ & $115 \quad(305-1141$ & \\
\hline & 2003 & 45.1 & $(41.6-48.6)$ & $48.2(45.4-51.1)$ & $46.8(44.6-49.1)$ \\
\hline \multirow[t]{4}{*}{ Homes with a smoke alarm or detector } & 1997 & & & $58.2(57.2-59.1)$ & \\
\hline & 1998 & & & $64.0(63.0-65.0)$ & \\
\hline & 2002 & & & $72.9(71.8-74.0)$ & \\
\hline & 2003 & & & $72.7(71.6-73.9)$ & \\
\hline \multirow[t]{4}{*}{ Recommended daily fruit intake } & 1997 & 37.8 & $(36.4-39.2)$ & $51.1(49.8-52.4)$ & $44.5(43.6-45.5)$ \\
\hline & 1998 & 38.0 & $(36.5-39.5)$ & $49.2(47.9-50.5)$ & $43.7(42.7-44.7)$ \\
\hline & 2002 & 40.3 & $(38.4-42.2)$ & $50.1(48.4-51.7)$ & $45.3(44.0-46.5)$ \\
\hline & 2003 & 39.0 & $(37.1-40.9)$ & $52.4(50.8-54.0)$ & $45.8(44.6-47.1)$ \\
\hline \multirow[t]{4}{*}{ Recommended vegetable intake } & 1997 & 10.8 & $(10.0-11.7)$ & $21.7(20.6-22.7)$ & $16.3(15.6-17.0)$ \\
\hline & 1998 & 9.8 & $(8.9-10.6)$ & $20.5(19.5-21.6)$ & $15.2(14.5-15.9)$ \\
\hline & 2002 & 9.2 & $(8.2-10.3)$ & $22.9(21.6-24.2)$ & $16.2(15.3-17.0)$ \\
\hline & 2003 & 11.8 & $(10.7-12.9)$ & $26.7(25.3-28.0)$ & $19.3(18.4-20.3)$ \\
\hline \multirow[t]{4}{*}{ Usual use of low-fat, reduced fat or skim milk } & 1997 & 37.5 & $(36.0-38.9)$ & $53.8(52.4-55.1)$ & $45.7(44.7-46.7)$ \\
\hline & 1998 & 38.8 & $(37.3-40.3)$ & $52.4(51.1-53.8)$ & $45.7(44.7-46.7)$ \\
\hline & 2002 & 35.8 & $(34.0-37.6)$ & $50.7(49.0-52.4)$ & $43.4(42.1-44.6)$ \\
\hline & 2003 & 37.1 & $(35.2-38.9)$ & $50.8(49.2-52.4)$ & $44.0(42.8-45.3)$ \\
\hline \multirow[t]{2}{*}{ Food insecurity last 12 months } & 2002 & 5.2 & $(4.4-6.0)$ & $6.1 \quad(5.3-6.9)$ & $5.7 \quad(5.1-6.2)$ \\
\hline & 2003 & 5.3 & $(4.5-6.2)$ & $6.9 \quad(6.1-7.6)$ & $(5.5-6.7)$ \\
\hline \multirow[t]{3}{*}{ Adequate physical activity } & 1998 & 52.2 & $(50.7-53.7)$ & $43.1(41.8-44.4)$ & $47.6(46.6-48.6)$ \\
\hline & 2002 & 50.4 & $(48.4-52.3)$ & $42.9(41.2-44.5)$ & $46.6(45.3-47.8)$ \\
\hline & 2003 & 49.5 & $(47.6-51.5)$ & $40.6(39.0-42.1)$ & $45.0(43.7-46.2)$ \\
\hline \multirow[t]{4}{*}{ Current daily or occasional smoking } & 1997 & 27.2 & $(25.9-28.5)$ & $21.0(20.0-22.0)$ & $24.0(23.2-24.9)$ \\
\hline & 1998 & 26.2 & $(24.8-27.5)$ & $21.3(20.2-22.4)$ & $23.7(22.9-24.6)$ \\
\hline & 2002 & 23.9 & $(22.2-25.6)$ & $18.9(17.6-20.2)$ & $21.4(20.3-22.4)$ \\
\hline & 2003 & 25.0 & $(23.3-26.8)$ & $20.0(18.7-21.3)$ & $22.5(21.4-23.6)$ \\
\hline \multirow[t]{4}{*}{ Smoke-free households } & 1997 & & & $69.8(68.9-70.6)$ & \\
\hline & 1998 & & & $73.2(72.3-74.1)$ & \\
\hline & 2002 & & & $81.0(80.0-82.0)$ & \\
\hline & 2003 & & & $82.5(81.6-83.4)$ & \\
\hline Hand washing when preparing raw meat & 2003 & 56.3 & $(54.2-58.5)$ & $64.4(62.9-65.9)$ & $60.8(59.5-62.0)$ \\
\hline
\end{tabular}

Source: NSW Health Survey 1997, 1998, 2002, and 2003 (HOIST), Centre for Epidemiology and Research, NSW Department of Health 
information covering limitation of daily activities, and bodily pain experienced in the previous 4 weeks, was collected. These indicators will continue to be monitored.

\section{HEALTH SERVICES}

As part of the continuing commitment to monitoring satisfaction with health services in NSW, questions were asked about the use of and satisfaction with a range of services. These included difficulties with getting health care when needed, admission to hospital, attending an emergency department, using community health centres, and using public dental services.

Overall, there was a significant increase in the proportion of people who reported having difficulties getting health care when needing it between 1997 and 2003 (10.0 per cent to 13.3 per cent) (Table 3 ).
There were no changes in the proportion of people who gave positive ratings of hospital inpatient care (91.2 per cent) and emergency department care (78.9 per cent) between 1997 and 2003. While the proportion of people giving positive ratings of public dental care increased between 2002 and 2003 (81.2 per cent to 85.4 per cent), the increase was not significant.

Emergency department attendance in the previous 12 months (13.5 per cent) and hospital admission in the previous 12 months (13.5 per cent) both remained unchanged between 1997 and 2003, as did public dental service attendance in the previous 12 months (4.3 per cent) between 2002 and 2003. The proportion of people attending a comunity health centre in the previous 12 months decreased between 2002 and 2003 (6.9 per cent to 5.1 per cent).

\section{TABLE 2}

TRENDS IN INDICATORS OF HEALTH STATUS, BY SEX, NSW, 1997-2003

\begin{tabular}{|c|c|c|c|c|c|}
\hline Indicator & Year & Males & $(95 \% \mathrm{Cl})$ & Females $(95 \% \mathrm{Cl})$ & Persons $(95 \% \mathrm{CI})$ \\
\hline \multirow[t]{4}{*}{$\begin{array}{l}\text { Excellent, very good, or good self-rated } \\
\text { health status }\end{array}$} & 1997 & 84.9 & $(83.9-85.8)$ & $85.0(84.1-85.9)$ & $84.9(84.3-85.6)$ \\
\hline & 1998 & 84.9 & $(83.9-85.9)$ & $83.0(82.1-83.9)$ & $83.9(83.2-84.6)$ \\
\hline & 2002 & 81.8 & $(80.3-83.3)$ & $79.7(78.5-81.0)$ & $80.7(79.7-81.7)$ \\
\hline & 2003 & 81.9 & $(80.5-83.3)$ & $79.8(78.5-81.0)$ & $80.8(79.9-81.7)$ \\
\hline \multirow[t]{4}{*}{ Ever diagnosed with asthma } & 1997 & 14.9 & $(13.9-16.0)$ & $18.1(17.1-19.2)$ & $16.6(15.8-17.3)$ \\
\hline & 1998 & 15.4 & $(14.3-16.5)$ & $18.0(17.0-19.0)$ & $16.7(16.0-17.5)$ \\
\hline & 2002 & 18.3 & $(16.8-19.9)$ & $20.9(19.6-22.3)$ & $19.6(18.6-20.7)$ \\
\hline & 2003 & 19.4 & $(17.8-21.0)$ & $22.7(21.4-24.0)$ & $21.0(20.0-22.1)$ \\
\hline \multirow[t]{4}{*}{ Current asthma } & 1997 & 8.7 & $(7.9-9.5)$ & $11.9(11.0-12.8)$ & $10.3 \quad(9.7-10.9)$ \\
\hline & 1998 & 8.9 & $(8.0-9.8)$ & $10.9(10.1-11.7)$ & $9.9 \quad(9.3-10.5)$ \\
\hline & 2002 & 9.1 & $(8.0-10.2)$ & $12.0(11.0-13.0)$ & $10.6 \quad(9.8-11.3)$ \\
\hline & 2003 & 9.2 & $(8.0-10.3)$ & $12.7(11.7-13.7)$ & $11.0(10.2-11.7)$ \\
\hline \multirow[t]{4}{*}{ Diabetes or high blood sugar } & 1997 & 5.2 & $(4.6-5.8)$ & $4.3 \quad(3.8-4.8)$ & $4.7 \quad(4.3-5.1)$ \\
\hline & 1998 & 4.9 & $(4.2-5.5)$ & $(3.5-4.5)$ & $(4.0-4.9)$ \\
\hline & 2002 & 6.6 & $(5.8-7.4)$ & $(5.0-6.4)$ & $(5.6-6.7)$ \\
\hline & 2003 & 6.9 & $(6.1-7.8)$ & $5.5 \quad(4.9-6.2)$ & $(5.7-6.7)$ \\
\hline \multirow[t]{4}{*}{ High and very high psychological distress } & 1997 & 9.2 & $(8.4-10.0)$ & $12.9(12.0-13.8)$ & $11.1(10.5-11.7)$ \\
\hline & 1998 & 9.0 & $(8.1-9.9)$ & $12.0(11.1-12.8)$ & $10.5 \quad(9.9-11.1)$ \\
\hline & 2002 & 10.5 & $(9.3-11.6)$ & $14.0(12.8-15.1)$ & $12.2(11.4-13.1)$ \\
\hline & 2003 & 9.3 & $(8.2-10.4)$ & $12.9(11.8-14.0)$ & $11.1(10.3-11.9)$ \\
\hline \multirow[t]{3}{*}{ All natural teeth missing } & 1998 & 5.8 & $(5.2-6.4)$ & $10.7(10.0-11.4)$ & $8.3 \quad(7.8-8.8)$ \\
\hline & 2002 & 5.2 & $(4.6-5.9)$ & $8.6 \quad(7.8-9.4)$ & $(6.4-7.5)$ \\
\hline & 2003 & 4.2 & $(3.6-4.8)$ & $7.4 \quad(6.8-8.0)$ & $5.8 \quad(5.4-6.2)$ \\
\hline \multirow[t]{4}{*}{ Overweight and obesity } & 1997 & 49.7 & $(48.3-51.2)$ & $34.5(33.3-35.8)$ & $42.2(41.2-43.1)$ \\
\hline & 1998 & 50.3 & $(48.7-51.8)$ & $34.5(33.2-35.7)$ & $42.5(41.4-43.5)$ \\
\hline & 2002 & 53.9 & $(52.0-55.9)$ & $38.5(36.9-40.1)$ & $46.3(45.0-47.6)$ \\
\hline & 2003 & 55.6 & $(53.7-57.6)$ & $41.0(39.4-42.6)$ & $48.3(47.1-49.6)$ \\
\hline \multirow[t]{4}{*}{ Obese } & 1997 & 11.1 & $(10.2-12.0)$ & $11.5(10.7-12.3)$ & $11.3(10.7-11.9)$ \\
\hline & 1998 & 12.6 & $(11.6-13.6)$ & $11.6(10.8-12.4)$ & $12.1(11.5-12.7)$ \\
\hline & 2002 & 14.8 & $(13.4-16.2)$ & $14.4(13.3-15.6)$ & $14.6(13.7-15.5)$ \\
\hline & 2003 & 15.5 & $(14.2-16.8)$ & $16.5(15.4-17.7)$ & $16.0(15.1-16.9)$ \\
\hline Fall in the last 12 months & 2003 & 18.7 & $(16.0-21.5)$ & $27.5(24.9-30.1)$ & $23.5(21.7-25.4)$ \\
\hline Incontinence in the last 4 weeks & 2003 & 11.2 & $(9.8-12.6)$ & $31.9(30.1-33.7)$ & $21.8(20.6-23.0)$ \\
\hline
\end{tabular}

Source: NSW Health Survey 1997, 1998, 2002, and 2003 (HOIST), Centre for Epidemiology and Research, NSW Department of Health 
TABLE 3

TRENDS IN INDICATORS OF USE AND SATISFACTION WITH HEALTH SERVICES, BY SEX, NSW, 1997-2003

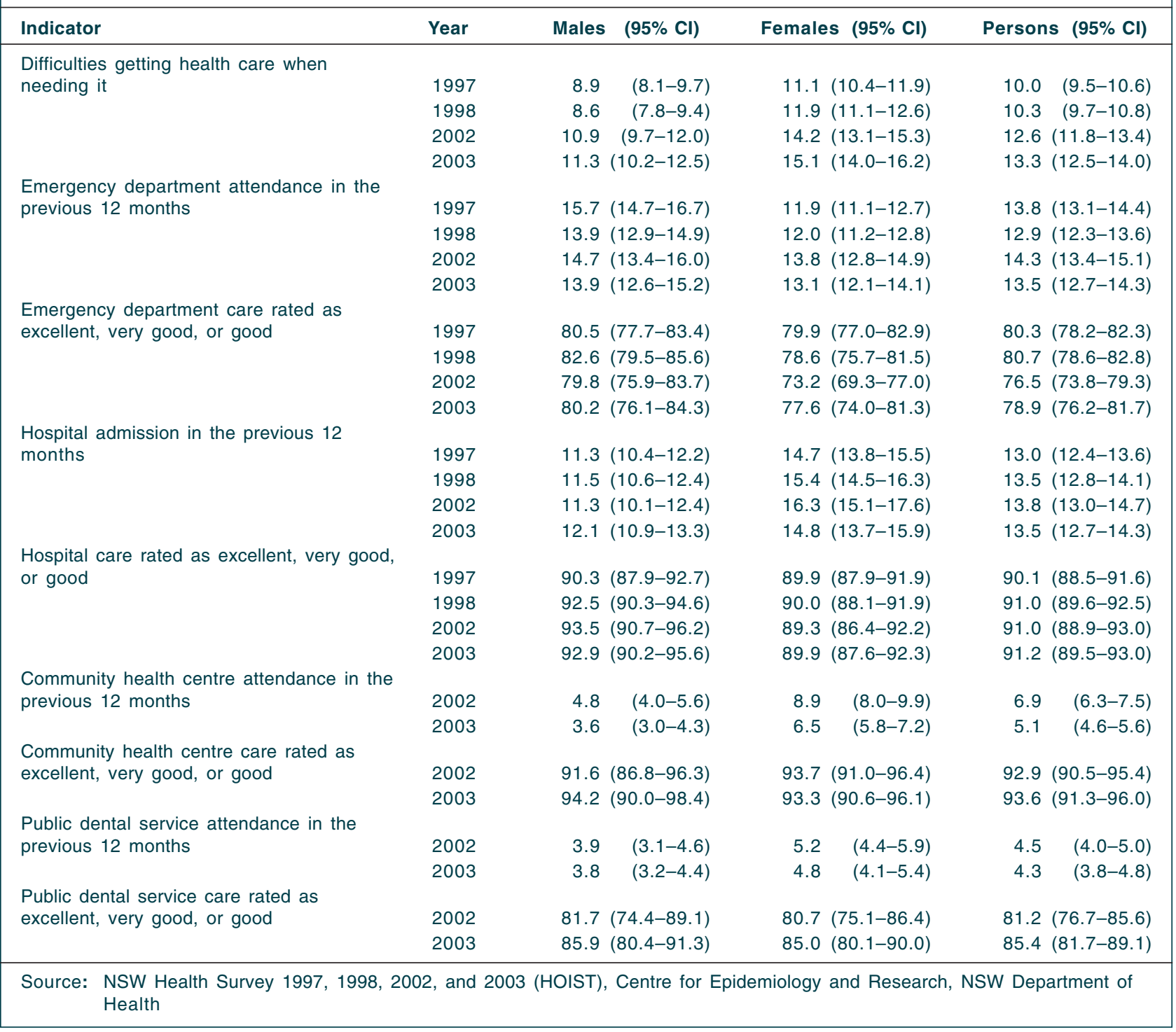

\section{SOCIAL CAPITAL}

The term 'social capital' refers to the institutions, relationships, and norms that shape social networks, foster trust, and facilitate coordination and cooperation for mutual benefit. The New South Wales Continuous Health Survey included questions on social reciprocity and neighbourhood connection, feelings of trust and safety, and participation in the local community. Between 2002 and 2003 there was no change in any of the indicators of social capital (Table 4).

\section{THE FUTURE}

There are a number of changes for the 2004 Continuous Health Survey. In the health status section, an expanded module on diabetes (focusing on complications and screening) will be included. Under health behaviours, cancer screening (mammographic, bowel, and cervical) will be included again along with rate of hysterectomy. In addition a module on summer sun protection and shade policy will be included.

In addition to these changes, there are new modules on interpersonal safety and violence in young adults aged 18-25 years, and on sight and hearing.

The New South Wales Continuous Health Survey provides information that will assist health professionals, health planners and those involved in policy development to plan, implement and evaluate health programs and initiatives within the community and within population and target groups. 


\section{TABLE 4}

TRENDS IN INDICATORS OF SOCIAL CAPITAL, BY SEX, NSW, 1997-2003

\begin{tabular}{|c|c|c|c|c|c|}
\hline Indicator & Year & Males & $(95 \% \mathrm{Cl})$ & Females $(95 \% \mathrm{Cl})$ & Persons $(95 \% \mathrm{Cl})$ \\
\hline \multirow[t]{2}{*}{$\begin{array}{l}\text { Attended a community event at least once in } \\
\text { the last } 6 \text { months }\end{array}$} & 2002 & 52.9 & $(51.0-54.9)$ & $60.5(58.9-62.1)$ & $56.8(55.5-58.0)$ \\
\hline & 2003 & 54.1 & $(52.2-56.0)$ & $62.0(60.4-63.5)$ & $58.1(56.9-59.3)$ \\
\hline \multirow[t]{2}{*}{$\begin{array}{l}\text { Helped out any local group or organisation } \\
\text { at least once in the past } 3 \text { months }\end{array}$} & 2002 & 30.5 & $(28.7-32.2)$ & $35.7(34.1-37.3)$ & $33.1(32.0-34.3)$ \\
\hline & 2003 & 31.2 & $(29.4-33.0)$ & $32.9(31.4-34.4)$ & $32.1(30.9-33.2)$ \\
\hline \multirow[t]{2}{*}{$\begin{array}{l}\text { Active member of a local organisation, } \\
\text { church or club }\end{array}$} & 2002 & 45.5 & $(43.6-47.5)$ & $42.3(40.7-43.9)$ & $43.9(42.6-45.1)$ \\
\hline & 2003 & 45.4 & $(43.5-47.4)$ & $41.7(40.1-43.3)$ & $43.5(42.3-44.8)$ \\
\hline \multirow[t]{2}{*}{ Most people can be trusted } & 2002 & 69.0 & $(67.2-70.8)$ & $62.9(61.3-64.6)$ & $65.9(64.7-67.2)$ \\
\hline & 2003 & 71.5 & $(69.7-73.2)$ & $67.9(66.3-69.4)$ & $69.6(68.5-70.8)$ \\
\hline \multirow[t]{2}{*}{ Feel safe walking down their street after dark } & 2002 & 78.0 & $(76.4-79.5)$ & $55.8(54.2-57.5)$ & $66.8(65.6-67.9)$ \\
\hline & 2003 & 80.2 & $(78.8-81.7)$ & $56.6(55.1-58.2)$ & $68.3(67.2-69.4)$ \\
\hline \multirow[t]{2}{*}{ Area has a reputation for being a safe place } & 2002 & 75.2 & $(73.6-76.9)$ & $71.6(70.1-73.1)$ & $73.4(72.3-74.5)$ \\
\hline & 2003 & 76.5 & $(74.8-78.1)$ & $73.1(71.7-74.5)$ & $74.8(73.7-75.9)$ \\
\hline \multirow[t]{2}{*}{ Visit neighbours } & 2002 & 68.7 & $(66.9-70.5)$ & $63.2(61.6-64.8)$ & $65.9(64.7-67.1)$ \\
\hline & 2003 & 67.0 & $(65.2-68.9)$ & $63.8(62.3-65.4)$ & $65.4(64.2-66.6)$ \\
\hline \multirow[t]{2}{*}{$\begin{array}{l}\text { Able to ask for neighbourhood help to care } \\
\text { for a child }\end{array}$} & 2002 & 73.3 & $(71.5-75.1)$ & $68.0(66.4-69.6)$ & $70.6(69.4-71.8)$ \\
\hline & 2003 & 74.2 & $(72.4-76.0)$ & $71.9(70.5-73.4)$ & $73.0(71.9-74.2)$ \\
\hline \multirow[t]{2}{*}{$\begin{array}{l}\text { Run into friends and acquaintances when } \\
\text { shopping in local area }\end{array}$} & 2002 & 80.4 & $(78.8-82.0)$ & 83.7 (82.4-84.9) & $82.0(81.1-83.0)$ \\
\hline & 2003 & 80.3 & $(78.8-81.9)$ & $82.8(81.6-84.1)$ & $81.6(80.6-82.6)$ \\
\hline \multirow[t]{2}{*}{ Sad to leave neighbourhood } & 2002 & 71.2 & $(69.4-73.0)$ & 75.7 (74.3-77.2) & $73.5(72.4-74.7)$ \\
\hline & 2003 & 69.4 & $(67.5-71.2)$ & $76.8(75.4-78.2)$ & $73.1(72.0-74.3)$ \\
\hline
\end{tabular}

Source: NSW Health Survey 1997, 1998, 2002, and 2003 (HOIST), Centre for Epidemiology and Research, NSW Department of Health

Printed copies of the New South Wales Adult Health Survey 2003 are available from the NSW Health Survey Program on (02) 9424 5707. Electronic copies can be downloaded in PDF format from the NSW Department of Health's website at www.health.nsw.gov.au/public-health/phb/phb.html, and in HTML format from www.health.nsw.gov.au/public-health/survey/hsurvey.html. 